\title{
Die Bestimmung der Schwefelsäure als Bariumsulfat bei Gegenwart von Aluminium.
}

\author{
Von L. Moser und P. Kour. \\ Mit 1 Figur im Text.
}

Die Unregelmäßigkeiten, welche bei der Fällung des Sulfations mit Bariumchlorid in Gegenwart verschiedener Stoffe, wie FerriAluminium- und Chromiion auftreten, waren schon seit langem die Ursache des Studiums dieser Frage durch viele Forscher. Dabei wurde hauptsächlich dem Ferriion besondere Aufmerksamkeit gewidmet, da dieses im Hinblick auf die Schwefelbestimmung im Pyrit von großer Bedeutung ist. Seit den grundlegenden Arbeiten von LungE ${ }^{1}$ ) und KÜster und ThIEL ${ }^{2}$ ) wurde dieses Thema sowohl vom theoretischen, wie auch vom rein analytischen Standpunkte behandelt, wobei nicht nur die Ansichten über die Theorie, sondern auch die Verbesserungsvorschläge vielfach einander widersprechen, was bei der schwierigen Behandlung dieser Aufgabe nicht zu verwundern ist. So stellen Küster und ThIEL die Behauptung auf, daß Ferrisulfat mitgefällt werde, welche Auffassung schon früher $J_{\text {ANNASCH }}$ und RICHARDs ${ }^{3}$ ) ausgesprochen hatten. Bereits im darauffolgenden Jahre änderten Küster und ThIEL ${ }^{4}$ ) ihre Meinung dahin, daB sie die Bildung eines Bariumsalzes der Ferrischwefelsäure als Ursache der zu niedrigen Ergebnisse der Schwefelsäurebestimmung annehmen. Gegen diese Ansicht trat RIChands ${ }^{5}$ ) auf, der den Vorgang als Okklusion auffaBt, und in einer anderen Abhandlung kommt SchNeIDeR ${ }^{6}$ ) zu dem Schlusse, dab hier eine feste Lösung vorliege, da die im Bariumsulfatniederschlage befindlichen Eisenmengen proportional dem Gehalt der Lösung an Ferriion ansteigen.

1) Lonae, Z. angew. Chem. 1892, 447.

2) Küster and ThuEL, Z. anorg. Chem. 19 (1899), 97.

3) JanNasch und Richands, Journ, prakt. Chem. [2] 39 (1889), 321.

4) Küster und ThIEL, Z. anorg. Chem. 22 (1900), 424.

$\left.{ }^{5}\right)$ Richards, Z. anorg. Chem. 23 (1900), 383.

B) Schnmider, Z. physik. Chem. 10 (1892), 425. 
VAN'т Krujis ${ }^{1}$ ) wieder meint, daB das Mitreißen der Sulfate mehr chemischen als physikalischen Charakter trüge, indes Jonnstow und $\mathrm{ADAMS}^{2}$ ) das gerade Gegenteil behaupten und von einer reinen Adsorptionserscheinung sprechen. Jeder dieser Autoren begründet natürlich seine Ansicht ausführlich, ohne daß es bisher zu einer Einigung gekommen wäre. Dieses Resultat kann nicht verwundern, wenn man bedenkt, daß es sich hier um relativ kleine Substanzmengen und anderseits um verwickelte Vorgänge handelt, denen man weder mit den rein analytischen noch mit chemischphysikalischen Hilfsmitteln beikommen kann.

Neben dem Ferrion trat naturgemäß das Aluminiumion stark in den Hintergrund, obgleich es von Interesse hätte sein müssen, einen Vergleich zwischen diesen beiden analytisch so ähnlichen Elementen im Hinblick auf ihr Verhalten gegen ausfallendes Bariumsulfat ziehen zu können. Mit dem Aluminium befaßte sich in einer besonderen Arbeit bisher bloß Creighton ${ }^{3}$ ), auf welche noch zurückgekommen werden wird, da ihre Ergebnisse im Widerspruch mit unserer Untersuchung zu stehen scheinen. Einige Sätze widmet KaraOglanow ${ }^{4}$ ) dem Aluminium in einer Abhandlung über die gewichtsanalytische Bestimmung der Schwefelsäure, die darin gipfeln, daß dieses Element dabei keinen Fehler verursache, was, wie wir zeigen werden, nicht zutreffend ist. Einige Bemerkungen finden sich schließlich in Arbeiten von WINKLER ${ }^{5}$ ) und von KolthofF und Vogelenzang. ${ }^{6}$ )

Bevor nun die eigenen Versuche beschrieben werden, sei kurz die Art und Weise der Ausführung der Analysen angegeben, so daß später nicht mehr darauf zurückgekommen zu werden braucht. Es sei noch betont, daß nur mit chemisch reinen Stoffen gearbeitet wurde, die auf das Vorhandensein bestimmter Verunreinigungen untersucht worden waren.

Es wurde eine bestimmte Menge Schwefelsäure (oder Aluminiumsulfatlösung) von genau bekanntem Titer mit $200 \mathrm{ccm}$ Wasser (größere Verdünnung erwies sich als ohne Einfluß auf das Ergebnis) verdünnt, n/1-Salzsäure und Aluminiumchlorid in einer der Absicht

\footnotetext{
1) VAN'T KRUJS, $Z$. analyt. Chem. 49 (1910), 393.

2) JoHnston and Adams, Journ. Am. Chem. Soc. 33 (1911), 829.

3) Creighton, Z. anorg. Chem. 63 (1909), 53.

4) Karaoglanow, $Z$. analyt. Chem. 56 (1917), 417.

5) L. W. WINKLER, Z. angew. Chem. a3 (1920), 59.

4) Konthofe and Vogmeanzang, Z. analyt. Chem. 58 (1919), 49.
} 
des Versuches entsprechenden Weise zugegeben, zum Kochen erhitzt and die Fällung mit 10\% iger Bariumchloridlösung tropfenweise, unter stetem Umrühren vorgenommen; die Fällungsdauer betrug meist 4-5 Minuten. Nach vollendeter Fällung wurde noch einige Minuten gekocht, nach 12-24 Stunden mit heißem Wasser dekantiert, filtriert und chlorfrei gewaschen. Das Filter wurde meist noch feucht eingeäschert und samt dem Niederschlag im Platintiegel über dem Teclubrenner 20 Minuten geglüht bis zur Gewichtskonstanz.

Die Bestimmung des Aluminiums im Bariumsulfat geschah durch AufschluB mit reinstem Natriumkarbonat, die Schmelze wurde mit verdünnter Salzsäure ausgelaugt, filtriert, aufgekocht, und in der Platinschale das Aluminium nach Zugabe von etwas Chlorammon mit kohlensäurefreiem Ammoniak gefällt und als $\mathrm{Al}_{2} \mathrm{O}_{3}$ zur Wägung gebracht.

Bei den Versuchen wurden folgende Lösungen verwendet:

$\mathrm{H}_{2} \mathrm{SO}_{4}$ ungefähr 0,17 molar; $50 \mathrm{ccm} \ldots 0,7960 \mathrm{~g} \mathrm{BaSO}$, $\mathrm{AlCl}_{3}$ ungefähr 0,10 molar; $1 \mathrm{ccm} \ldots .0,00245 \mathrm{~g} \mathrm{AlCl}_{3}$, $\mathrm{HCl}$ ungefähr 0,10 molar.

1. Versuchsreihe.

Tabelle 1.

Gleiche Mengen Schwefelsäure, Wasser, Salzsäure, steigende Mengen Aluminiumchlorid.

\begin{tabular}{c|c|c|c|c|c|c}
\hline $\begin{array}{c}\text { Versuch } \\
\mathrm{Nr}\end{array}$ & $\begin{array}{c}\mathrm{H}_{2} \mathrm{SO}_{4} \\
\mathrm{ccm}\end{array}$ & $\begin{array}{c}\mathrm{H}_{2} \mathrm{O} \\
\mathrm{ccm}\end{array}$ & $\begin{array}{c}\mathrm{n} / \mathrm{l}-\mathrm{HCl} \\
\mathrm{ccm}\end{array}$ & $\begin{array}{c}\mathrm{AlCl}_{3} \\
\mathrm{ccm}\end{array}$ & $\begin{array}{c}\mathrm{BaSO}_{4} \\
\mathrm{~g}\end{array}$ & $\begin{array}{c}\mathrm{Al}_{2} \mathrm{O}_{3} \mathbf{m g} \\
\mathrm{im} \mathrm{Ba}_{2} \mathrm{SO}_{4}\end{array}$ \\
\hline $\mathbf{1}$ & 50 & 200 & 5 & 0 & 0,7960 & 0,00 \\
2 & 50 & 200 & 5 & 10 & 0,7953 & 0,60 \\
3 & 50 & 200 & 5 & 20 & 0,7948 & - \\
4 & 50 & 200 & 5 & 25 & 0,7928 & - \\
5 & 50 & 200 & $\mathbf{5}$ & $\mathbf{4 0}$ & 0,7921 & - \\
6 & 50 & 200 & $\mathbf{5}$ & 50 & 0,7925 & 0,80 \\
7 & 50 & 200 & 5 & 100 & 0,7923 & 0,90 \\
8 & 50 & 200 & 5 & 200 & 0,7934 & - \\
9 & 50 & 200 & 5 & 300 & 0,7925 & -
\end{tabular}

Tabelle 2.

Gleiche Mengen Schwefelsäure, Wasser, keine Salzsäure, steigende Mengen $\mathrm{AlCl}_{3}$.

\begin{tabular}{|c|c|c|c|c|c|c|}
\hline $\begin{array}{c}\text { Versuch } \\
\text { Nr. }\end{array}$ & $\begin{array}{c}\mathrm{H}_{2} \mathrm{SO}_{4} \\
\mathrm{ccm}\end{array}$ & $\begin{array}{l}\mathrm{H}_{2} \mathrm{O} \\
\mathrm{com}\end{array}$ & $\begin{array}{c}\text { n/l-HCl } \\
\mathrm{ccm}\end{array}$ & $\begin{array}{c}\mathrm{AlCl}_{3} \\
\mathrm{ccm}\end{array}$ & $\begin{array}{c}\mathrm{BaSO}_{4} \\
\mathrm{~g}\end{array}$ & $\begin{array}{r}\mathrm{Al}_{2} \mathrm{O}_{3} \mathrm{mg} \\
\mathrm{im} \mathrm{BaSO}_{4}\end{array}$ \\
\hline $\begin{array}{l}10 \\
11 \\
12 \\
13\end{array}$ & $\begin{array}{l}50 \\
50 \\
50 \\
50\end{array}$ & $\begin{array}{l}200 \\
200 \\
200 \\
200\end{array}$ & $\begin{array}{l}- \\
-\end{array}$ & $\begin{array}{r}0 \\
20 \\
50 \\
100\end{array}$ & $\begin{array}{l}0,7963 \\
0,7941 \\
0,7911 \\
0,7906\end{array}$ & $\begin{array}{l}0,00 \\
0,20 \\
0,11 \\
0,19\end{array}$ \\
\hline
\end{tabular}


Tabelle 3.

Gleiche Mengen Schwefelsäure, Wasser, Salzsäure, steigende Mengen Aluminiumchlorid.

\begin{tabular}{c|c|c|c|c|c|c}
\hline $\begin{array}{c}\text { Versuch } \\
\text { Nr. }\end{array}$ & $\begin{array}{c}\mathrm{H}_{2} \mathrm{SO} \mathrm{O}_{4} \\
\mathrm{ccm}\end{array}$ & $\begin{array}{c}\mathrm{H}_{2} \mathrm{O} \\
\mathrm{ccm}\end{array}$ & $\begin{array}{c}\mathrm{n} / \mathrm{l} \cdot \mathrm{HCl} \\
\mathrm{ccm}\end{array}$ & $\begin{array}{c}\mathrm{AlCl}_{3} \\
\mathrm{ccm}\end{array}$ & $\begin{array}{c}\mathrm{BaSO}_{4} \\
\mathrm{~g}\end{array}$ & $\begin{array}{c}\mathrm{Al}_{2} \mathrm{O}_{3} \mathrm{mg} \\
\mathrm{im} \mathrm{BaSO}\end{array}$ \\
\hline 14 & 50 & 200 & 20 & 0 & 0,7951 & 0,00 \\
15 & 50 & 200 & 20 & 20 & 0,7959 & 0,00 \\
16 & 50 & 200 & 20 & 50 & 0,7949 & 0,10 \\
17 & 50 & 200 & 20 & 100 & 0,7932 & 0,14
\end{tabular}

Tabelle 4.

Gleiche Mengen Schwefelsäure, Wasser, Alùminiumchlorid, steigende Mengen Salzsäure.

\begin{tabular}{c|c|c|c|c|c|c}
\hline $\begin{array}{c}\text { Versuch } \\
\mathrm{Nr} .\end{array}$ & $\begin{array}{c}\mathrm{H}_{2} \mathrm{SO}_{4} \\
\mathrm{ccm}\end{array}$ & $\begin{array}{c}\mathrm{H}_{2} \mathrm{O} \\
\mathrm{ccm}\end{array}$ & $\begin{array}{c}\mathrm{n} / \mathrm{l}-\mathrm{HCl} \\
\mathrm{ccm}\end{array}$ & $\begin{array}{c}\mathrm{AlCl}_{3} \\
\mathrm{ccm}\end{array}$ & $\begin{array}{c}\mathrm{BaSO}_{4} \\
\mathrm{~g}\end{array}$ & $\begin{array}{c}\mathrm{Al}_{2} \mathrm{O}_{3} \mathrm{mg} \\
\mathrm{im} \mathrm{BaSO}_{4}\end{array}$ \\
\hline$=-18$ & 50 & 200 & 0 & 50 & 0,7911 & 1,8 \\
19 & 50 & 200 & 5 & 50 & 0,7925 & 0,80 \\
20 & 50 & 200 & 20 & 50 & 0,7949 & 0,10 \\
21 & 50 & 200 & 40 & 50 & 0,7952 & - \\
22 & 50 & 200 & 60 & 50 & 0,7948 & 0,13
\end{tabular}

Aus den Ergebnissen der in den Tabellen 1-4 verzeichneten Versuche ist zu ersehen, daß die Anwesenheit von Aluminiumion erniedrigend auf die Auswage an Bariumsulfat wirkt und zwar zunächst um so stärker, je mehr Aluminiumchlorid zugegen ist. Von einer bestimmten Menge Aluminium angefangen, bleibt jedoch der gefundene Wert an Bariumsulfat konstant (Versuche 4-9) und beträgt der maximale Fehler $-0,5 \%$ des richtigen Bariumsulfatresultates.

Daß aber auch die zugesetzte Menge Salzsäure das Ergebnis innerhalb gewisser Grenzen beeinflußt, kann man aus den Tabellen 2 bis 4 ersehen. In neutraler Lösung ist dieser negative Fehler am größten (Versuch 10-13) und in stark salzsaurer Lösung (Versuch 14 bis 17, 20-22) wird er kleiner, was durch die mit wachsender Salzsäurekonzentration verknüpfte erhöhte Einschließung von Bariumchlorid bedingt ist, wodurch ein teilweiser Ausgleich erfolgt.

Die im Bariumsulfat eingeschlossenen Mengen Aluminiumoxyd sind jedenfalls relativ gering und von einer bestimmten Grenze an konstant, unabhängig von der Menge des vorhandenen Aluminiumions.

Stellt man die Ergebnisse der Tab. 1 graphisch dar (Fig. 1), wobei die Abszissen die Anzahl Kubikzentimeter des zugesetzten 
Alnminiumchlorids, die Ordinaten die Auswagen an Bariumsulfat in Gramm bedeuten, so erkennt man, daß die Kurve vom Punkte 4 an ungefähr parallel der Abszissenachse verläuft, daß also von $\mathrm{BaSO}_{45}$

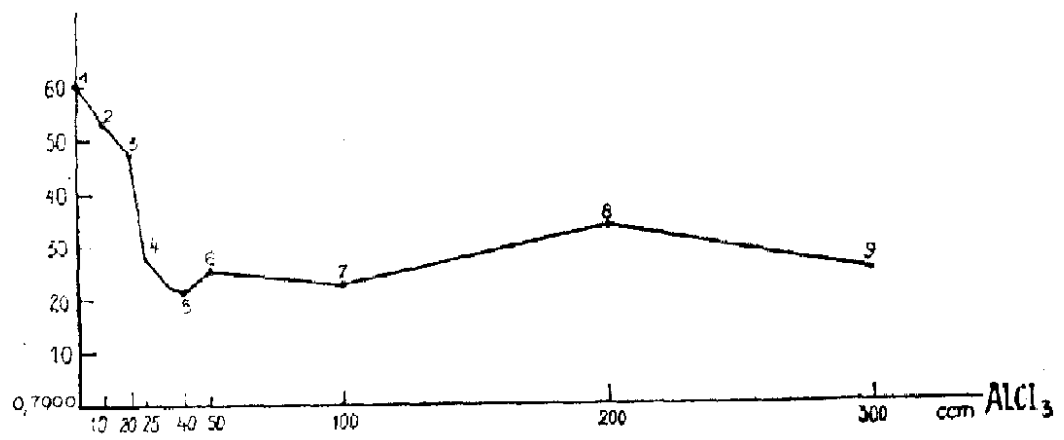

Fig. 1.

hier an diesteigenden Mengen von Aluminiumion keine weitere Verminderung des Wertes an Bariumsulfat ergeben.

\section{Versuchsreihe.}

Unter Einhaltung derselben Versuchsbedingungen wurde die Fällung der Schwefelsaure in Cegenwart einer elwa 0,09 molaron Ferrichloridlösung vorgenommen.

Tabelle 5 .

\begin{tabular}{c|c|c|c|c|c|c}
\hline $\begin{array}{c}\text { Versuch } \\
\mathrm{Nr} .\end{array}$ & $\begin{array}{c}\mathrm{H}_{2} \mathrm{SO}_{4} \\
\mathrm{ccm}\end{array}$ & $\begin{array}{c}\mathrm{H}_{2} \mathrm{O} \\
\mathrm{ccm}\end{array}$ & $\begin{array}{c}\mathrm{n} / 1-\mathrm{HCl} \\
\mathrm{ccm}\end{array}$ & $\begin{array}{c}\mathrm{FlCl}_{3} \\
\mathrm{ccm}\end{array}$ & $\begin{array}{c}\mathrm{BaSO}_{4} \\
\mathrm{~g}\end{array}$ & $\begin{array}{c}\mathrm{Fe}_{2} \mathrm{O}_{\mathrm{a}} \mathrm{mg} \\
\mathrm{im} \mathrm{BaSO}_{4}\end{array}$ \\
\hline $\mathbf{2 3}$ & $\mathbf{5 0}$ & 200 & 5 & 0 & 0,7960 & 0,0 \\
$\mathbf{2 4}$ & 50 & 200 & 5 & 10 & 0,7809 & 8,0 \\
25 & 50 & 200 & 5 & 20 & 0,7819 & 8,9 \\
26 & 50 & 200 & 5 & 50 & Hydrolysiert beim Kochen
\end{tabular}

Tabelle 6.

\begin{tabular}{c|c|c|c|c|c|c}
\hline $\begin{array}{c}\text { Versuch } \\
\mathrm{Nr} .\end{array}$ & $\begin{array}{c}\mathrm{H}_{2} \mathrm{SO}_{4} \\
\mathrm{cem}\end{array}$ & $\begin{array}{c}\mathrm{H}_{2} \mathrm{O} \\
\mathrm{cem}\end{array}$ & $\begin{array}{c}\mathrm{n} / 1-\mathrm{HCl} \\
\mathrm{cem}\end{array}$ & $\begin{array}{c}\mathrm{FeCl}_{3} \\
\mathrm{com}\end{array}$ & $\begin{array}{c}\mathrm{BaSO}_{4} \\
\mathrm{~g}\end{array}$ & $\begin{array}{c}\mathrm{Fe}_{2} \mathrm{O}_{3} \mathrm{mg} \\
\mathrm{im} \mathrm{BaSO}_{4}\end{array}$ \\
\hline $\mathbf{2 7}$ & 50 & 200 & 20 & 0 & 0,7951 & 0,0 \\
28 & 50 & 200 & 20 & 10 & 0,7842 & 4,3 \\
29 & 50 & 200 & 20 & 20 & 0,7843 & 6,2 \\
30 & 50 & 200 & 20 & 50 & 0,7831 & 6,4 \\
31 & 50 & 200 & 20 & 100 & 0,7826 & 7,5 \\
$\mathbf{3 2}$ & 50 & 200 & 60 & 50 & 0,7883 & 2,2
\end{tabular}


Das Eisen zeigt genau dasselbe Verhalten, nur sind hier die negativen Fehler um eine ganze Zehnerpotenz, also wesentlich größer, auch hier bleibt von einer bestimmten Grenze an die aufgenommene Menge Ferrioxyd annähernd dieselbe.

Es handelt sich nun darum, unsere Ergebnisse mit jenen Creightons ${ }^{1}$ ) in Zusammenhang zu bringen. Da sei vorweg bemerkt, daß Creighton nicht Schwefelsäure, sondern Ammonsulfat mit Bariumchlorid in Gegenwart von Aluminiumchlorid bestimmte. Er findet so, daß die Anwesenheit kleiner Mengen Al-Ions die Wirkung hätten, das Gewicht des Bariumsulfats wesentlich zu erhöhen, und übereinstimmend mit uns, daB steigende Mengen von Aluminiumchlorid einem Grenzwerte im Hinblicke auf die Aufnahme durch das Bariumsulfat zustreben.

Nun ist auf den ersten Blick nicht einleuchtend, warum das Aluminium bei Anwendung von Ammonsulfat positive, bei Schwefelsäure negative Fehler hervorrufen soll, denn aus der Tatsache allein, daß bei der Fällung von Ammonsulfat ein niedrigeres, als das theoretische Resultat erhalten wird (so geben z. B. Aluen und Johnstos ${ }^{2}$ ) das Fehlermaximum mit $-0,75 \%$ an) folgt noch nicht, daB Aluminium, das ebenfalls ein zu niedriges Ergebnis liefert, hier eine Erhöhung bewirkt. Nun kommt aber das zu niedrige Resultat bei der Fällung von Ammonsulfat dadurch zustande, daß etwas von diesem Salz in den Bariumsulfatniederschlag mitgeht und dieses verflüchtigt sich beim Glühen vollkommen. Das Aluminiumchlorid bewirkt offenbar durch Bildung des weniger dissoziierten Aluminiumsulfats eine Verminderung des vom Bariumsulfat aufgenommenen Ammonsulfats, indem es selbst aufgenommen wird, und dadurch kommt eine relative Erhöhung des Resultates zustande, oder mit anderen Worten, es wird der stark erniedrigende Einfluß des Ammonsulfats durch den weniger erniedrigenden Einfluß des Aluminiums abgeschwächt. Das erbaltene Resultat ist aber noch immer niedriger als der theoretisch richtige Wert.

Trotzdem also der störende EinfluB des Aluminiumions bei der Fällung der Schwefelsäure kein großer ist, wurde doch versucht, eine Trennung des Aluminiums von der Schwefelsäure zwecks Durchführung einer vollständig richtigen Bestimmung dieser letzteren vorzunehmen. Dabei können dreierlei Möglichkeiten in Betracht gezogen werden:

1) Creichton, 1. C.

2) Axund und Johnston, Joure. Am. Chem. S06. 32 (1910), 588. 
1. Fällung und Filtration des Al'--Ions und darauffolgende Bestimmung der Schwefelsäure im Filtrate.

2. Es verbleibt das gefüllte Al"'-Ion in der Flüssigkeit und wird erst nach durchgeführter $\mathrm{SO}_{4}{ }^{\prime \prime}$-Ionenfällung wieder in Lösung gebracht.

3. Überführung des $\mathrm{Al}^{\cdots}$-Ions in Komplexionen (oder Aluminat) und Fällung des $\mathrm{SO}_{4}{ }^{\prime \prime}$-Ions in derselben Lösung.

Zu 1. Der einfachste Weg wäre, das Al'-Ion mit Ammoniak zu fällen und im Filtrate die Schwefelsäure zu bestimmen, analog der LuNGaschen Methode für Ferrieisen. Das ist aber deshalb nicht möglich, weil das mit Ammoniak gefällte $\mathrm{Al}(\mathrm{OH})_{3}$ stets basische Aluminiumsulfate enthältt ${ }^{1}$ ), deren $\mathrm{SO}_{4}{ }^{\prime \prime}$-Ionen für die folgende Fällung der Schwefelsäure verloren gingen; auch ist bei etwas größeren Aluminiummengen das Auswaschen des Niederschlages sehr erschwert, man müßte ihn mehrmals lösen und wieder fällen. Dabei ist weiter noch nachteilig, da $B$ das nur etwas angetrocknete Aluminiumbydroxydgel sich nur äuBerst langsam in. Salzsäure löst, demnach verspricht dieser Weg keinen Erfolg.

Nach einer älteren Literaturangabe ${ }^{2}$ ) soll das Aluminiumhydroxyd in Gegenwart von Glyzerin nicht kolloidal, sondern flockig ausfallen, wodurch das Auswaschen erleichtert werden würde. Wir konnten uns durch eine Anzahl von unter verschiedenen Verhältnissen ausgeführten Versuchen überzeugen, daß der Niederschlag ebenso gallertig ausfällt. ${ }^{3}$ )

Zu 2. Hier kommt zunächst die für Eisen allgemein angewendete Methode von Küster und THIEL in Betracht. Die Lösung wurde in der Hitze mit Ammoniak versetzt (es zeigte sich, daß es nicht ron Belang ist, ob dieses im größeren Überschusse oder nur bis zum deutlichen Geruch danach zugegeben wird), dann die Fällung mit Bariumchlorid (sowohl tropfenweise, wie auch in einem Gusse) vorgenommen, nun mit Salzsäure angesäuert, einige Minuten aufgekocht und wie gewöhnlich weiter behandelt.

Das Verfahren ist bei tropfenweiser Fällung und Anwesenheit größerer Mengen Aluminium überhaupt undurchführbar, bei Fällung in einem Gusse liefert es unbrauchbare - zu hohe Resultate, die dadurch zustande kommen, daß viel Aluminium mitgewogen wird. Der Grund ist leicht einzusehen. Wie bereits erwähnt,

1) O. Sommatolia, Z, angew. Chem. 1903, 202.

2) GUYARd, Z. analyt. Chem. 22 (1883), 426.

3) Einzelheiten in der Dissertation von P. KoHx. 
fallen durch Ammoniak aus $\mathrm{Al}^{\cdots}$ - IIonenlösungen bei Gegenwart von $\mathrm{SO}_{4}{ }^{\prime \prime}$ stets basische Sulfate des Aluminiums mit. In diesem Falle ginge wohl kein $\mathrm{SO}_{4}{ }^{\prime \prime}$-Ion für die Sulfatlösung verloren, da diese Reste bei der folgenden Behandlung mit Salzsäure wieder in Lösung gehen und demnach vom im Überschuß vorhandenen $\mathrm{Ba}$ "-Ion gefällt werden. Dagegen haben die basischen Aluminiumsulfate jedoch die Eigenschaft, ihre Löslichkeit in verdünnten Säuren dann zu verringern, wenn sie längere Zieit auf höhere Temperatur erwärmt wurden, was bei der tropfenweisen Fällung in der Wärme unvermeidlich, aber auch bei der Fällung in einem Gusse nicht völlig zu umgehen ist. Im ersteren Falle erhält man einen Niederschlag von Bariumsulfat, der so stark mit basischen Aluminiumsalzen verunreinigt ist, daß diese die Dauer der Filtration wesentlich verlängern, in letzterem Falle enthält das geglühte Bariumsulfat ebenfalls noch viel Aluminium und die Ergebnisse fallen zu hoch aus.

Um die Bildung basischer Salze zu verhindern, wurde versucht, von der leichten Hydrolisierbarkeit von $\mathrm{Al}^{\prime}$-Ionen Gebrauch zu machen. Vorerst wurde die Nitritmethode nach ScHIRM ${ }^{1}$ ) angewendet, demnach wurde ein Teil der Säure mit einigen Tropfen Ammoniak neutralisiert (ohne daß ein Niederschlag ausfiel), mit der berechneten Menge Nitrit versetzt, mit Wasser verdünnt, so lange gekocht, bis keine Stickoxyde mehr vorhanden waren und dann erst die Fällung des $\mathrm{SO}_{4}{ }^{\prime \prime}$-Ions vorgenommen und nachher mit Salzsäure angesäuert.

Es wurden wieder $50 \mathrm{ccm} 0,17$ molare Schwefelsäure, $200 \mathrm{ccm}$ Wasser und $100 \mathrm{~cm} 0,09$ molare $\mathrm{AlCl}_{3}$-Lösung angewandt, die Nitritlösungen waren $10 \%$ ig; es wurde gefunden:

Vers. 33: $0,8082 \mathrm{~g}$ BaSO${ }_{4}$. Vers. 34: 0,8186 g $\mathrm{BaSO}_{4}$; berechnet $0,7960 \mathrm{~g} \mathrm{BaSO}_{4}$.

Die Ergebnisse fielen zu hoch aus, was auf die Okklusion des durch die Zersetzung der salpetrigen Säure entstehenden Nitrations zurückzuführen ist. Der Beweis für die Richtigkeit dieser Auffassung wird dadurch erbracht, daß Schwefelsäure ohne Zusatz von $\mathrm{AlCl}_{3}$ in derselben Weise behandelt, ebenfalls höhere Auswagen an Bariumsulfat gibt. Wir erhielten so einmal 0,8014 und $0,8025 \mathrm{~g} \mathrm{BaSO}_{4}$ (Vers. 35 und 36). Hier zeigt sich außerdem die Wirkung des mit dem Al'--Ion verbundenen Anions, wodurch mehr Salpetersäure gebildet wurde und das Resultat demgemäß höher ausfällt.

1) E. SCHIRM, Chem. Ztg. 33 (1909), 877. 
Zu 3. Zuerst warde die Fällung der Schwefelsäure aus der Aluminatlösung versucht, wobei das Chlorbarium in der Siedehitze sowohl tropfenweise, als auch auf einmal zugefügt wurde. Dann erst wurde mit Salzsäure angesäuert und sonst wie früher verfahren. Wir erhielten so stark erhöhte Resultate, das Bariumsulfat war aber weniger mit Aluminium, als mit Alkali verunreinigt. Auch die Überführung des $\mathrm{Al}^{\prime \prime}$-Ions in ein komplexes Ion mittels Oxalsäure oder Ammonoxalat wurde versucht; hier nähern sich wohl die erhaltenen Werte den theoretisch richtigen, vorausgesetzt, daß nur so viel Oxalation zugegeben wird, als zur Bindung des $\mathrm{Al}^{\cdots}$ Ions notwendig ist; durch einen Überschuß wird jedoch eine beträchtliche Erhöhung des Ergebnisses bewirkt, deren Ursache uns nicht bekannt ist. Allen aus alkalischer oder a mmoniakalischer Lösung gefällten $\mathrm{BaSO}_{4}$-Niederschlägen haftet überdies noch der Übelstand an, daß sie zufolge ihres außerordentlich feinkristallinischen Gefüges schwer filtrierbar sind.

Von Intersese war schließlich noch die „umgekehrte Fällung* durch Einfließenlassen einer $\mathrm{Al}_{2}\left(\mathrm{SO}_{4}\right)_{3}$-Lösung in die verdünnte $\mathrm{BaCl}_{2}$-Lösung. In ammoniakalischer Lösung zu arbeiten, erwies sich als unmöglich, weil auch hier schwer lösliche basische Aluminiumsalze ausfallen. Daher wurde so gearbeitet, daß die heiße, Aluminiumchlorid enthaltende Schwefelsäure in die auf $200 \mathrm{ccm}$ verdünnte, mit $20 \mathrm{ccm} \mathrm{n} / 1$-Salzsäure angesäuerte Bariumchloridlösung langsam einfließen gelassen wurde. Wie die untenstehenden Zahlen zeigen, fiel auch hier der Wert an Bariumsulfat zu hoch aus; es wurde wieder $\mathrm{Al}_{2} \mathrm{O}_{3}$ im Niederschlag nachgewiesen, übrigens findet so auch erhöhte Okklusion von Bariumchlorid statt.

Angewandt wurden $50 \mathrm{ccm} \mathrm{H}_{2} \mathrm{SO}_{4}$, entsprechend 1,5010 $\mathrm{g} \mathrm{BaSO}_{4}$, $20 \mathrm{ccm} \mathrm{n} / 1 \cdot \mathrm{HCl}, 200 \mathrm{ccm} \mathrm{H}_{2} \mathrm{O}$ und $15 \mathrm{cem} \mathrm{BaCl}_{2}$ von $10 \%$.

Vers. 35. Zusatz: $50 \mathrm{cem} 0,09 \mathrm{~mol} . \mathrm{AlCl}_{3}$, gef. 1,5164 $\mathrm{g} \mathrm{BaSO}_{4}$ und $0,0037 \mathrm{~g} \mathrm{Al}_{2} \mathrm{O}_{3}$,

Vers. 36. Zusatz: $100 \mathrm{ccm} 0,09 \mathrm{~mol} . \mathrm{AlCl}_{3}$, gef. $1,5170 \mathrm{~g} \mathrm{BaSO}_{4}$ und $0,0035 \mathrm{~g} \mathrm{Al}_{2} \mathrm{O}_{3}$.

Auf Grund unserer Versuche können wir demnach die günstigsten Bedingungen für die Fällung der Schwefelsäure bei Gegenwart von Aluminiumion in nachfolgender Arbeitsvorschrift zusammenfassen:

Die Lösung, welche ein Volumen von $250-300 \mathrm{ccm}$ (pro $1 \mathrm{~g}$ Bariumsulfat) einnimmt, wird mit $20 \mathrm{ccm}$ n/1-Salzsäure versetzt, 
zum Sieden erhitzt und tropfenweise ein geringer ÜberschuB an 10\% iger heißer Bariumchloridlösung zugefügt, nach beendeter Fällung wird einige Minuten aufgekocht und unter Anwendung von aufgeschlämmtem Filterpapier filtriert, mit heißem Wasser chlorfrei ausgewaschen und der noch feuchte Niederschlag samt dem Filter im Platintiegel 20 Minuten über der vollen Flamme eines guten Brenners stark geglüht.

Die Auswage an Bariumsulfat ist selbst bei Anwesenheit größerer Mengen Aluminium höchstens um $0,15 \%$ zu niedrig.

Dieses Ergebnis steht im Einklange mit den Erfahrungen Winklers'), der bei der Bestimmung der Schwefelsäure im Alaun bei Verwendung von $5 \mathrm{ccm}$ Salzsäure statt des theoretischen Wertes von $40,49 \% \mathrm{SO}_{4}$ nur $40,27 \%$ fand. Unrichtig dagegen ist die Erklärung von Kolthoff und Vocelenzasa ${ }^{2}$ ), die angeben, daB dabei der positive Fehler des Aluminiums durch den negativen Fehler des Kaliums teilweise ausgeglichen wird. Es hat hier vielmehr dieselbe Erklärung zu gelten, welche als Deutung der von CReightos ${ }^{3}$ ) gefundenen Tatsachen schon oben aufgestellt wurde.

\section{Theoretisches.}

Es bleibt nur noch übrig, einige Worte über die Art des Vorganges zu sagen. Auf Grund unserer Versuche und theoretischer Überlegungen neigen wir zu der Annahme, daß hier ein ausgesprochener Fall einer festen Lösung vorliege. Dafür spricht, daß die Verunreinigung des Bariumsulfats mit Aluminiumoxyd bzw. Aluminiumsulfat nur bis zu einer bestimmten Grenze möglich ist; ist die Sättigung des Bariumsulfates mit dem Aluminiumsalz einmal erreicht, so findet eine vermehrte Verunreinigung des Niederschlages durch steigende Mengen von Aluminiumchlorid, die der Lösung weiterhin zugefügt werden, nicht mehr statt. Graphisch ist dies auch aus dem Verlauf der Kurve (Fig. 1), die der Tab. 1 entspricht, zu ersehen. Während die ersten zugesetzten Mengen Aluminiumachlorid eine stark zunehmende Verunreinigung des Niederschlages bewirken und die Kurve steil abwärts verläuft, trifft dies vom Punkte 5 an, der $40 \mathrm{ccm} \mathrm{AlCl}_{3}$ entspricht, nicht mehr zu, von da an ist ein im Prinzip zur Abszissenachse paralleler Verlauf zu erkennen, woran selbst der große Zusatz von $300 \mathrm{ccm} \mathrm{AlCl}_{3}$ nichts ändert. Es liegt

1) WINKr.ER, $Z$. angew. Chem. 30 (1917), 254.

9) Kolthorf und Vogridanzana, 1. c.

3) Crmahtow, 1 . c. 
voraussichtlich eine feste Lösung von Aluminiumsulfat im Bariumsulfat vor, was sich auch daraus ergibt, daB trotz der Aufnahme von Aluminium das Resultat erniedrigt wird, ein Vorgang, der nur durch die Bildung von Aluminiumsulfat erklärt werden kann. Der Verlauf einer derartigen Kurve ist außerdem charakteristisch für eine feste Lösung. Dafür spricht auch, daß nicht nur das Bariumsulfat im Entstehungszustande die Eigenschaft hat, Aluminiumsalz, wie wir uns überzeugen konnten, aufzunehmen, sondern, auch das fertig gebildete, in verdünnter Salzsäure aufgeschlämmte Produkt zeigt genau dieselben Eigenschaften, es nimmt ebensoviel Aluminiumoxyd auf. Damit ist die Ansicht von Kuing and Lassieur ${ }^{1}$ ) widerlegt, die meinen, daß das sogenannte „Mitreißen" von Aluminium nur durch das Bariumsulfat im Entstehungszustande erfolge. Übrigens haben die Versuche von PATten $^{2}$ ) das gleiche Verhalten des Bariumsulfats gegenüber den Chloriden von Eisen, Chrom, Mangan, Nickel und Kobalt ergeben. Patten ist der Ansicht, daß ,die betreffenden Metalle das fertig gebildete Bariumsulfat durchdringen und sich in dasselbe einbetten", was ja ziemlich gleichbedeutend mit der Annahme einer festen Lösung ist. Gerade der Umstand, daß fertig gebildetes Bariumsulfat sich ebenso verhält gegen das Aluminiumion, wie wenn es erst entstünde, spricht gegen die Bildung einer chemischen Verbindung, denn es ist schwer zu glauben, daß das praktisch unlösliche Bariumsulfat, wenn es sich nicht gerade im statu nascendi befindet, so reaktionsfähig sein sollte.

SchlieBlich wäre noch zu erwähnen, daß sowohl nach unseren, wie auch nach den Versuchen von SCHNEIDER ${ }^{3}$ ) das Ferriion ein durchwegs ähnliches Verhalten zeigt, auch hier erreicht die im Bariumsulfat eingeschlossene Menge einen bestimmten Grenzwert.

\section{Zusammenfassung.}

1. Es wurde gezeigt, daß die Fällung der Schwefelsäure bei Gegenwart von Aluminium mit einem negativen Fehler behaftet ist, und daß die Verunreinigung des Bariumsulfates durch Aluminium. sulfat zuerst proportional dem in der Lösung befindlichen Aluminiumion ansteigt, von einer bestimmten Grenze an aber unabhängig ist von weiter ansteigenden Aluminiummengen.

1) Kling und lassiaur, C. R. 158 (1914), 487.

2) Patten, Journ. Am. Chem. Soc. 95 (1903), 186.

3) SChNetder, Z. physik. Chem. 10 (1892), 425. 
310 C. Moser und P. Kohn. Bestimmung der Schwefelsäure als Bariumsulfat.

2. Der durch Aluminium verursachte negative Fehler ist bei geeigneter Arbeitsweise relativ klein, er ist rund um eine Zehnerpotenz kleiner, als der durch Ferriion bewirkte analoge Fehler.

3. Es wurden erfolglos verschiedene Trennungsverfahren versucht, um obigen Fehler auszuschalten.

4. Als beste Bestimmungsmethode ergibt sich die Fällung der Schwefelsäure bei Gegenwart von Aluminiumion in salzsaurer Lösung und wurde nach diesem Grundsatz eine Arbeitsvorschrift gegeben, wobei das Maximum des negativen Fehlers - 0,15\% beträgt.

5. Es wurde gezeigt, daß nicht nur Bariumsulfat im Entstehungszustande, sondern auch ein schon gefälltes Produkt Aluminiumsulfat aufzunehmen vermag und daraus und aus dem unter 1 . Gesagten geschlossen, daß man es hier mit der Bildung einer festen Lösung von Aluminiumsulfat im Bariumsulfat zu tun habe.

Wien, Laboratorium für analytische Chemie der Technischen Hochschule.

Bei der Redaktion eińgegangen am 30. März 1922. 